Discrete Comput Geom 38:15-28 (2007)

DOI: $10.1007 / \mathrm{s} 00454-007-1324-9$

\title{
A Positive Semidefinite Approximation of the Symmetric Traveling Salesman Polytope
}

\author{
Ellen Veomett \\ Department of Mathematics, University of Michigan, \\ Ann Arbor, MI 48109, USA \\ eveomett@umich.edu
}

\begin{abstract}
For a convex body $B$ in a vector space $V$, we construct its approximation $P_{k}, k=1,2, \ldots$, using an intersection of a cone of positive semidefinite quadratic forms with an affine subspace. We show that $P_{k}$ is contained in $B$ for each $k$. When $B$ is the Symmetric Traveling Salesman Polytope on $n$ cities $T_{n}$, we show that the scaling of $P_{k}$ by $n / k+O(1 / n)$ contains $T_{n}$ for $k \leq\lfloor n / 2\rfloor$. Membership for $P_{k}$ is computable in time polynomial in $n$ (of degree linear in $k$ ). We also discuss facets of $T_{n}$ that lie on the boundary of $P_{k}$ and we use eigenvalues to evaluate our bounds.
\end{abstract}

\section{Introduction and Results}

For many interesting convex bodies $X$ in a vector space $V$ along with a point $x \in V$, the question "is $x$ in $X$ ?" is difficult to answer. This fact has generated work in the direction of finding another set $Y$ which is "close" to $X$ in some way for which the membership question is "easy" to answer. Sherali and Adams [11], Lovász and Schrijver [9], and Lasserre [7] have constructed approximating sets in the case where the body to be approximated is a $0-1$ polytope. In each of these instances, the authors constructed successive relaxations of a $0-1$ polytope, such that in the $n$th step, the $0-1$ polytope is achieved: $P=K^{n} \subset K^{n-1} \subset \cdots \subset K^{1} \subset K$. For specifics, as well as a comparison of the methods, see [8]. Kojima and Tunçel [6] have similarly behaving constructions for a general convex body $F$. Given a first approximation $C_{0} \supset F$, they create successive approximations $C_{k}$ such that $F \subset C_{k} \subset C_{k-1}$ and $F=\cap_{i=0}^{\infty} C_{i}$. Metric properties are not known for each of the aforementioned constructions.

In the following we construct successive relaxations of an arbitrary convex body $X$, each of which is contained in $X$. If $X \subset \mathbb{R}^{n}$ is a 0-1 polytope, then we also obtain $P_{n}=X$ (for details see Section 1.3). We explore in particular the case where $X$ is the Symmetric Traveling Salesman Polytope, where we estimate the closeness of the approximation metrically. 


\subsection{The Symmetric Traveling Salesman Polytope}

The Symmetric Traveling Salesman Polytope (STSP) can be described as follows: recall that a Hamiltonian cycle in the complete graph on $n$ vertices $K_{n}$ is a cycle which visits every vertex exactly once. To each Hamiltonian cycle in $K_{n}$, we can associate its incidence matrix $A=\left(a_{i j}\right)$ where

$$
a_{i j}= \begin{cases}1 & \text { if the cycle contains edge }\{i, j\} \\ 0 & \text { if the cycle does not contain edge }\{i, j\} .\end{cases}
$$

Note that each matrix corresponding to a Hamiltonian cycle is a symmetric 0-1 matrix in $\mathbb{R}^{n^{2}}$ with zeros on the diagonal. Given a particular matrix corresponding to a Hamiltonian cycle, any other such matrix can be obtained from it by simultaneously permuting rows and columns (this corresponds to permuting the labels on the vertices of the graph). The STSP is the convex hull of all adjacency matrices corresponding to Hamiltonian cycles in $K_{n}$. Thus the vertices of the STSP are each a matrix which corresponds to a cycle. To each cycle we can associate a permutation of the numbers $\{1,2, \ldots, n\}$ beginning with the number 1 where the permutations $\left(1, m_{2}, m_{3}, \ldots, m_{n}\right)$ and $\left(1, m_{n}, m_{n-1}, \ldots, m_{2}\right)$ are identified. We will use the descriptions of the vertices as matrices, cycles, and permutations interchangeably. Using the permutation description of a Hamiltonian cycle, it is not hard to see that there are $(n-1) ! / 2$ different Hamiltonian cycles in $K_{n}$.

The STSP has been studied widely, though a complete description of it via linear inequalities is not known (and in some sense, cannot be known unless NP $=$ co-NP, see [5]). It is clearly not full-dimensional in $\mathbb{R}^{n^{2}}$, being the convex hull of symmetric matrices with zeros on the diagonal. It is not hard to show that its dimension is $n(n-3) / 2$ for $n \geq 3$. For more information on the STSP and the associated Traveling Salesman Problem, see, for example, Chapter 58 of [10]. Some analysis of metric properties of inequalities for the related Graphic Traveling Salesman Problem can be found in [2]. Linear optimization over the STSP and the membership question for the STSP are known to be NP-hard.

\subsection{Semidefinite Construction}

The following observation of Barvinok [1] gives the construction with which we will work. Let $V$ be a real vector space and let $X \subset V$ be a finite (though possibly very large) set. Let $V^{*}$ denote the dual of $V$. Recall that the polar dual of $X$ is the set

$$
X^{\circ}=\{f: X \rightarrow \mathbb{R}: f \text { is linear, } f(x) \leq 1 \text { for all } x \in X\} \subset V^{*} .
$$

We note that by " $f$ linear" we mean that $f$ is the restriction to $X$ of a linear function on $V$. We view $X^{\circ}$ as living in the space $\mathbb{R}^{X}$ of all functions from $X$ to $\mathbb{R}$. If the convex hull of $X$ does not contain the origin in its relative interior, $X^{\circ}$ is not bounded. Indeed, one can find a linear function $f \in V^{*}$ not identically zero on all of $X$ which separates the origin and $X(f(x) \leq 0, \forall x \in X)$ so that $\alpha f \in X^{\circ}$ for all nonnegative $\alpha$. We thus consider the polar of $X$ in its affine span with the center of polarity being the barycenter 
of $X$ :

$$
A=\left\{f: X \rightarrow \mathbb{R}: f \text { is affine, } f(x) \leq 1 \text { for all } x \in X, \frac{1}{|X|} \sum_{x \in X} f(x)=0\right\} .
$$

Again, we note that by " $f$ affine" we mean that $f$ is the restriction to $X$ of an affine function on $V$. Then for convenience, we flip $A \mapsto-A$ and then shift $f \mapsto f+1$ so that we obtain the following description of the dual:

$$
Q=\left\{f X \rightarrow \mathbb{R}: f \text { is affine, } f(x) \geq 0 \text { for all } x \in X, \frac{1}{|X|} \sum_{x \in X} f(x)=1\right\} .
$$

The set with which we will work is $Q$.

We note that any convex body $B$ can be written as the polar dual to some other convex body $B^{\prime}$ which is in the same affine span, with the center of polarity the barycenter of $B^{\prime}$. Since $B^{\prime}$ can be arbitrarily closely approximated by a convex hull of finitely many points, $B$ is arbitrarily close to some $Q$ as defined above.

Fix a positive integer $k$ and let $\mathcal{P}_{k}(V)$ be the space of all polynomials of degree at most $k$ on $V$. To any function $f: X \rightarrow \mathbb{R}$ we can associate the quadratic form

$$
q_{f}: \mathcal{P}_{k}(V) \rightarrow \mathbb{R}
$$

defined by

$$
q_{f}(h)=\frac{1}{|X|} \sum_{x \in X} f(x) h^{2}(x) \quad \text { for } \quad h \in \mathcal{P}_{k}(V) .
$$

Clearly, if $f(x) \geq 0$ for each $x \in X$, then $q_{f}$ is a positive semidefinite quadratic form on $\mathcal{P}_{k}(V)$.

Note that, as $f$ ranges over affine functions on $X$ with average value 1 , the form $q_{f}$ ranges over an affine subspace in the space of quadratic forms on $\mathcal{P}_{k}(V)$. We define $\mathcal{A}_{k}$ to be this affine subspace, and define $\mathcal{K}_{k}$ to be the cone of positive semidefinite quadratic forms $q: \mathcal{P}_{k}(V) \rightarrow \mathbb{R}$. We define $P_{k}=\left\{f: q_{f} \in \mathcal{A}_{k} \cap \mathcal{K}_{k}\right\}$. Then we can see that

$$
Q \subset P_{k} \quad \text { and } \quad P_{k+1} \subset P_{k} \text {, }
$$

which leads us to ask

How close is $P_{k}$ to $Q$ ?

\subsection{The Case of a 0-1 Polytope}

We note that if $X \subset \mathbb{R}^{n}$ consists of 0 - 1 vectors, then $P_{n}=Q$. Indeed, let $f \in P_{n}$ so that $q_{f}$ is a positive semidefinite quadratic form and $f$ corresponds to an affine function with average value 1 on $X$. Let us fix any $y \in X$. Let $I \subset\{1,2, \ldots, n\}$ consist of the indices of the entries of $y$ which are 0 , and $J \subset\{1,2, \ldots, n\}$ be the indices of the entries of $y$ which are 1 . Then the degree $n$ polynomial

$$
p_{y}(x)=\prod_{i \in I}\left(1-x_{i}\right) \prod_{j \in J} x_{j}
$$


has value 1 on $y$ and 0 on any other vector in $X$. Thus, we have

$$
0 \leq q_{f}\left(p_{y}\right)=\frac{1}{|X|} \sum_{x \in X} f(x) p_{y}^{2}(x)=\frac{f(y)}{|X|} .
$$

Since $y$ was arbitrary, we see that $f(x) \geq 0$ for each $x \in X$, so that $f \in Q$, giving us $P_{n} \subset Q$. Since we already had $Q \subset P_{n}$, we see that indeed $Q=P_{n}$.

\subsection{The Case of the STSP}

From this point on, we fix $X$ to be the set of matrices corresponding to Hamiltonian cycles in $K_{n}$, so that our vector space is $\mathbb{R}^{n^{2}}$. We introduce the scalar product on the space of $n \times n$ matrices:

$$
\langle X, Y\rangle=\sum_{i, j} x_{i j} y_{i j}
$$

The barycenter of the STSP is the matrix $Z=\left(z_{i j}\right)$ where

$$
z_{i j}= \begin{cases}\frac{2}{n-1} & \text { if } \quad i \neq j, \\ 0 & \text { if } \quad i=j,\end{cases}
$$

and the average value of any affine function on $X$ is simply its value on $Z$. Note that the all ones function: $\mathbb{l}(x)=1$ for all $x \in X$ can be written as a linear function, since in the affine span of $X$ it corresponds to the inner product with the vector $(1 / 2 n, 1 / 2 n, \ldots, 1 / 2 n)$. Thus, if $f$ is a linear function and $a \in \mathbb{R}$, then in the affine span of $X$ the affine function $f(x)+a$ is equal to the linear function $f(x)+a \rrbracket(x)$. Hence we can see that in the set

$$
A=\left\{f: X \rightarrow \mathbb{R}: f \text { is affine, } f(x) \leq 1 \text { for all } x \in X, \frac{1}{|X|} \sum_{x \in X} f(x)=0\right\}
$$

as defined in Section 1.2, we can actually have each $f$ being linear. Flipping $A^{\prime} \mapsto-A^{\prime}$ does not destroy linearity of the functions, nor does shifting $f \mapsto f+1$. Hence, in this case,

$$
Q=\left\{f: X \rightarrow \mathbb{R}: f \text { is linear, } f(x) \geq 0 \text { for all } x \in X, \frac{1}{|X|} \sum_{x \in X} f(x)=1\right\} .
$$

We define $P_{k}$ just as in Section 1.2, so that $P_{k}$ is the set of all linear functions $f$ with average value 1 on $X$ whose corresponding quadratic form $q_{f}$ is positive semidefinite:

$$
0 \leq q_{f}(p)=\frac{1}{|X|} \sum_{x \in X} f(x) p^{2}(x) \quad \text { for polynomials } p(x) \text { on } \mathbb{R}^{n^{2}} .
$$

Note that the function 1 is the center of $Q$. We have the following:

Theorem 1. For any $n \geq 9$ and any $k=1,2, \ldots,\lfloor n / 2\rfloor$, we have

$$
Q-1 \subset P_{k}-1 \subset\left(\frac{n}{k}+\frac{10}{n}\right)(Q-1) \text {. }
$$


Recall that by our definition of $Q$, the traveling salesman polytope $T_{n}$ is the set of points in the affine span of $X$ such that $f(x)-1 \geq-1$ for all $f \in Q$. Thus, defining $R_{k}$ to be the set of points in the affine span of $X$ such that $g(x)-1 \geq-1$ for all $g \in P_{k}$, and denoting the barycenter of $T_{n}$ by $Z$, we have

Corollary 1. Let $T_{n}$ be the symmetric traveling salesman polytope. For any $n \geq 9$ and any $k=1,2, \ldots,\lfloor n / 2\rfloor$, we have

$$
R_{k}-Z \subset T_{n}-Z \subset\left(\frac{n}{k}+\frac{10}{n}\right)\left(R_{k}-Z\right) .
$$

Note that the approximation gives us an upper bound on how far $P_{k}$ is from $T_{n}$. We also note that approximating the STSP with respect to its center allows us to solve the STSP approximately with respect to the average value. Specifically, suppose that $f$ is a linear objective function on the STSP. Then using Theorem 1, we can bound the difference between the optimal value and average value of $f$ on $T_{n}$ based on the difference between the optimal value of $f$ on $P_{k}$ and the average value of $f$ on $T_{n}$.

Let $C$ be the cone of positive semidefinite quadratic forms on a vector space $V$. Then membership in $C$ is decidable in time of order $\operatorname{dim}(V)^{3}$ (see, for example Chapter 1 of [4]). In the case where $V=\mathcal{P}_{k}\left(\mathbb{R}^{n^{2}}\right), \operatorname{dim}(V)=\left(\begin{array}{c}n^{2}+k \\ k\end{array}\right)$ so that membership in $K_{k}$ is decidable in time of order $n^{6 k}$.

The remainder of the paper is structured as follows: in Section 2 we discuss facets of the STSP which we know lie on the boundary of $P_{k}$, in Section 3 we use eigenvalues to discuss the quality of our approximation, and in Section 4 we prove the bounds in Theorem 1.

\section{Facets on the Boundary}

Although there is no known complete description of the STSP as a system of linear inequalities, many facets are known (see, for example, chapter 58 of [10]). Some wellknown facet defining inequalities are the following:

$$
\begin{aligned}
& 0 \leq x_{i j} \leq 1 \quad \text { for each } i, j, \\
& \sum_{\substack{j \in U \\
i \in V-U}} x_{i j} \geq 2 \quad \text { for each } U \subset V \text { with } \emptyset \neq U \neq V, \\
& \sum_{j \in U} x_{i j}-\sum_{\{k, \ell\} \in F} x_{k \ell} \geq 1-|F| \quad \begin{array}{l}
\text { for } U \subset V, \quad F \text { matching, } \\
|F| \geq 3 \text { odd, each edge of }
\end{array} \\
& \underset{i \in V-U}{i, j\} \notin F} \quad F \text { having one endpoint in } U \text {. }
\end{aligned}
$$

The functions corresponding to (1) are facets for $n \geq 5$ [3], and the functions corresponding to (2) (called the Subtour Elimination Constraints) are facets for $2 \leq|U| \leq|V|-2$ (see Section 58.5 of [10]). The functions corresponding to (3) are all facets for $F$ as specified (see Section 58.7 of [10]). 
Any facet of the STSP can defined by some linear inequality $f(x) \geq 0$ which is unique up to a scaling factor. If we scale so that the average value on $X$ is 1 , then we know that the scaled function must be in $P_{k}$. A natural question to ask would be: which (if any) of the linear functions defining a facet for the STSP lie on the boundary of $P_{k}$ ?

Let $h_{i j}$ be the linear function such that $h_{i j}(x) \geq 0$ corresponds to the right-hand side of inequality (1) for edge $\{i, j\}$. Consider the degree 1 polynomial $p_{i j}=x_{i j}$. Then $h_{i j}(x)$ is 0 whenever $x$ contains the edge $\{i, j\}$, and $p_{i j}(x)$ is 0 whenever $x$ does not contain the edge $\{i, j\}$. Thus, we have

$$
q_{h_{i j}}\left(p_{i j}\right)=\frac{1}{|X|} \sum_{x \in X} h_{i j}(x)\left(p_{i j}(x)\right)^{2}=0
$$

so that $h_{i j}$ lies on the boundary of $P_{k}$ for any $k \geq 1$. If we let $h_{i j}^{\prime}$ be the polynomial corresponding to the left-hand side of inequality (1) for the edge $\{i, j\}$, then we can again easily see

$$
q_{h_{i j}^{\prime}}\left(1-p_{i j}\right)=\frac{1}{|X|} \sum_{x \in X} h_{i j}^{\prime}(x)\left(1-p_{i j}(x)\right)^{2}=0
$$

so that $h_{i j}^{\prime}$ lies on the boundary of $P_{k}$ for any $k \geq 1$.

Now suppose that $h_{U}$ is a linear function such that $h_{U}(x) \geq 0$ corresponds to the facet (2) for some $U \subset V, \emptyset \neq U \neq V$. Without loss of generality, say $\min \{|U|,|V \backslash U|\}=$ $|U|=\ell$ and let $U=\left\{m_{1}, m_{2}, \ldots, m_{\ell}\right\}$. Consider the degree $\ell-1$ polynomial

$$
p_{U}=x_{m_{1} m_{2}} x_{m_{2} m_{3}} \cdots x_{m_{\ell-1} m_{\ell}} .
$$

Note that $h_{U}(x)=0$ whenever $x$ has exactly two edges going out of $U$, and $p_{U}(x) \neq 0$ only if $x$ contains the path $m_{1}, m_{2}, \ldots, m_{\ell}$, which implies that there are exactly two edges going out of $U$. Thus, we have

$$
q_{h_{U}}\left(p_{U}\right)=\frac{1}{|X|} \sum_{x \in X} h_{U}(x) p_{U}^{2}(x)=0
$$

so that $h_{U}$ is on the boundary of $P_{k}$ if $\min \{|U||V \backslash U|\}-1 \leq k$.

In general, suppose we have a linear function $f \geq 0$ that defines a facet. Then if we can construct a polynomial of degree $\leq k$ such that any cycle for which that polynomial is nonzero must be on the facet defined by $f, f$ is on the boundary of $P_{k}$. For example, consider $U$ and $F$ as in (3), say $U=\left\{m_{1}, m_{2}, \ldots, m_{\ell}\right\}$ and $F=$ $\left\{\left\{m_{1}, n_{1}\right\},\left\{m_{2}, n_{2}\right\}, \ldots,\left\{m_{2 s+1}, n_{2 s+1}\right\}\right\}(3 \leq 2 s+1 \leq \ell)$. Let $h_{U, F}$ be a linear function such that $h_{U, F} \geq 0$ defines the facet in (3) corresponding to $U$ and $F$. Define the degree $s+\ell$ polynomial $p_{U, F}(x)$ as follows:

$$
p_{U, F}(x)=\prod_{i=1}^{2 s+1} x_{m_{i} n_{i}} \prod_{j=1}^{s} x_{m_{2 j-1} m_{2 j}} \prod_{k=2 s+1}^{\ell-1} x_{m_{k} m_{k+1}} .
$$

Then whenever $p_{U, F}(x) \neq 0, x$ contains the paths $n_{1} m_{1} m_{2} n_{2}$, $n_{3} m_{3} m_{4} n_{4}, \ldots, n_{2 s-1} m_{2 s-1} m_{2 s} n_{2 s}, n_{2 s+1} m_{2 s+1} m_{2 s+2} m_{2 s+3} \cdots m_{l}$. This implies that

$$
\sum_{\substack{j \in U \\ i \in V-U \\\{i, j\} \notin F}} x_{i j}=1
$$


and

$$
\sum_{\{k, \ell\} \in F} x_{k \ell}=2 s+1=|F|
$$

so that $h_{U, F}(x)=0$. Thus,

$$
q_{h_{U, F}}\left(p_{U, F}\right)=\frac{1}{|X|} \sum_{x \in X} h_{U, F}(x) p_{U, F}^{2}(x)=0
$$

so that $h_{U, F}$ is on the boundary of $P_{k}$ if $k \geq s+\ell$.

\section{Approximation Appraisal via Eigenvalues}

Looking at the bounds obtained from Theorem 1, we see that for the first approximation $P_{1}$, we have the following:

$$
Q-\mathbb{1} \subset P_{1}-1 \subset\left(n+\frac{10}{n}\right)(Q-1) .
$$

A natural question to ask is the following:

Can we find a scaling factor $a \ll n$ such that in fact we have $P_{1}-1 \subset a(Q-1)$ ?

This occurs precisely when, for every function $f$ on the boundary of $Q$, the function $a(f-1)$ lies on the boundary or outside of $P_{1}-1$. In terms of quadratic forms, this is precisely when the quadratic form $q_{h}$ for $h=a f+(1-a) \rrbracket$ has a 0 or negative eigenvalue. Thus our question is equivalent to the following:

Can we find an $a \ll n$ such that for every $f$ on the boundary of $Q$, the quadratic form $q_{h}$ for $h=a f+(1-a) 1$ has a 0 or negative eigenvalue?

If the boundary of $Q$ consisted solely of the Subtour Elimination Constraints (defined in (2)), the answer to the above question would be yes with $a=\sqrt{n}$. The calculations required for this claim are not presented here, but can be found in [12].

This fact has several possible implications. It could mean that the bound of $n$ from Theorem 1 is closer to the optimal bound, but that we need to look for functions on the boundary of $Q$ beyond the Subtour Elimination Constraints to see that this bound is necessary. Or it could mean that the bound of $\sqrt{n}$ is closer to the optimal bound and we have yet to find a way to prove this.

We note that there is a polynomial time separation algorithm for the subtour elimination constraints. Indeed, an $x \in \mathbb{R}^{n(n-1) / 2}$ satisfies the subtour elimination constraints if and only if the minimum cut for the complete graph with capacities corresponding to the entries of $x$ is at least 2. The author is unaware of any known lift constraints whose description is polynomial in $n$ and whose projection achieves the subtour elimination constraints. 


\section{Proofs of Metric Bounds}

Recall that from Section 1.2, we already have $Q \subset P_{k}$. Thus, the only question we must address is: how far is $P_{k}$ from $Q$ ? In other words, given a function $f \in P_{k}$, that is, a function defining a positive semidefinite quadratic form via

$$
q_{f}(h)=\frac{1}{|X|} \sum_{x \in X} f(x) h^{2}(x) \quad \text { for } \quad h \in \mathcal{P}_{k}\left(\mathbb{R}^{n^{2}}\right),
$$

where $f$ is a linear function with average value 1 on $X$, how negative can the values of $f$ on $X$ be? The following lemma gives us a bound:

Lemma 1. Fix $y \in X$ and $f \in P_{k}$ so that $f$ is a linear function with average value 1 on $X$ and $q_{f}$ is positive semidefinite. Suppose that we find polynomials $p_{1}, \ldots, p_{m}$ of degree $k$ such that $p_{i}$ takes on only values 0 or 1 and there exist positive constants $b_{k}<c_{k}$ such that for any $i, j \in\{1,2, \ldots n\}, i \neq j$,

$$
\sum_{x \in X:\{i, j\} \in x} \sum_{\ell=1}^{m} p_{\ell}(x)=\left\{\begin{array}{lll}
b_{k} & \text { if } & \{i, j\} \notin y, \\
c_{k} & \text { if } \quad\{i, j\} \in y .
\end{array}\right.
$$

Then

$$
-\frac{b_{k}(n-1)}{2\left(c_{k}-b_{k}\right)} \leq f(y) .
$$

Proof. Let $f \in P_{k}$ so that $f: X \rightarrow \mathbb{R}$ is linear function with average value 1 on $X$. Let us fix $y \in X$. For each $i<j$, we define the symmetric matrix $e_{i j}=\left(\varepsilon_{s t}\right)$ as follows:

$$
\varepsilon_{s t}=\left\{\begin{array}{ll}
1 & \text { if } s=i \\
0 & \text { otherwise. }
\end{array} \text { and } t=j \quad \text { or if } s=j \text { and } t=i,\right.
$$

Note that each $x \in X$ can be written as a sum of the matrices $e_{i j}(i<j)$ for which $\{i, j\}$ is an edge in $x$. Each $e_{i j}$ will appear in exactly $(n-2)$ ! different $x \in X$. Thus, the fact that $f$ has average 1 on $X$ tells us:

$$
\begin{aligned}
1 & =\frac{2}{(n-1) !} \sum_{x \in X} f(x) \\
& =\frac{2}{(n-1) !} \sum_{x \in X} f\left(\sum_{\{i, j\} \in x, i<j} e_{i j}\right) \\
& =\frac{2}{(n-1) !} \sum_{i<j} f\left(e_{i j}\right)(n-2) ! \\
& =\sum_{i<j} \frac{2}{n-1} f\left(e_{i j}\right)
\end{aligned}
$$

which gives us

$$
\frac{n-1}{2}-f(y)=\sum_{\{i, j\} \notin y} f\left(e_{i j}\right)
$$

for any particular $y \in X$. 
Since $f \in P_{k}$, the form $q_{f}$ is positive semidefinite, so for any polynomial $p(x)$ we can write the inequality

$$
0 \leq q_{f}(p)=\frac{2}{(n-1) !} \sum_{x \in X} f(x) p^{2}(x)
$$

which implies

$$
0 \leq \sum_{x \in X} f(x) p^{2}(x)=\sum_{\substack{x \in X \\ i<j \\\{i, j\} \in x}} f\left(e_{i j}\right) p^{2}(x)
$$

Now assuming we have $p_{i}$ as stated in Lemma 1 , for each $k$ we find that

$$
0 \leq \sum_{\substack{x \in X \\ i<j \\\{i, j\} \in x}} f\left(e_{i j}\right) p_{\ell}^{2}(x)=\sum_{\substack{x \in X \\ i<j \\\{i, j\} \in x}} f\left(e_{i j}\right) p_{\ell}(x) \quad \text { for } \quad \ell=1,2, \ldots, m
$$

so that using (4) we have

$$
\begin{aligned}
0 \leq \sum_{\substack{x \in X \\
i<j \\
\{i, j\} \in x}} \sum_{\ell=1}^{m} f\left(e_{i j}\right) p_{\ell}(x) & =c_{k} \sum_{\{i, j\} \in y} f\left(e_{i j}\right)+b_{k} \sum_{\{i, j\} \notin y} f\left(e_{i j}\right) \\
& =c_{k} f(y)+b_{k}\left(\frac{n-1}{2}-f(y)\right)
\end{aligned}
$$

which then implies

$$
-\frac{b_{k}(n-1)}{2\left(c_{k}-b_{k}\right)} \leq f(y) .
$$

We note that Lemma 1 only gives a bound on how negative a function $f \in P_{k}$ can be, if we can find polynomials $p_{i}$ satisfying the assumptions. It may be that, in fact, $f$ is entirely nonnegative. Picking a particular set of polynomials, we will prove the following:

Proposition 1. Let us fix $y \in X$ and $f \in P_{k}$. If $n$ is even, then

$$
-\frac{n}{k}+1-\frac{n(k-1)}{k\left(n^{2}-k n-3 n+k+3\right)} \leq f(y) .
$$

If $n$ is odd then

$$
-\frac{n}{k}+1-\frac{n(k-1)}{k\left(n^{2}-n k-4 n+4+2 k\right)} \leq f(y) .
$$

To complete the calculations required for Proposition 1, we need a lemma: 
Lemma 2. Let $\left(k_{1}, k_{2}, \ldots, k_{m}\right)$ be a partition of $k(k+m \leq n)$ and let $K_{n}$ be the complete graph on $n$ vertices. Let $p_{1}, p_{2}, \ldots, p_{m}$ be vertex disjoint paths in $K_{n}$ of length $k_{1}, \ldots, k_{m}$, respectively. Then the number of Hamiltonian cycles in $K_{n}$ containing all of paths $p_{1}, \ldots, p_{m}$ is

$$
2^{m-1}(n-k-1) !
$$

Proof. Note that the restriction $k+m \leq n$ assures us that it is possible to find disjoint paths in $K_{n}$ of lengths $k_{1}, \ldots, k_{m}$. Say path $p_{1}$ consists of vertices $v_{1}, v_{2}, \ldots, v_{\ell}$ with $v_{i}$ adjacent to $v_{i+1}$ for $1 \leq i \leq \ell-1$. Any cycle containing all of the paths $p_{1}, \ldots, p_{m}$ can be written uniquely as a sequence of the vertices, beginning with the sequence $v_{1} v_{2} \cdots v_{\ell}$ (i.e., beginning with the path $p_{1}$ in a designated orientation). Thinking of the remaining paths as blocks of vertices with two orientations and all other vertices not appearing in a path as blocks with a single orientation, we find that there are $2^{m-1}(n-k-1)$ ! ways of ordering and orienting the remaining blocks. Each of these orders and orientations corresponds uniquely to a Hamiltonian cycle containing paths $p_{1}, \ldots, p_{m}$.

Proof of Proposition 1. We use Lemma 1. First we need to describe the polynomials which we will use. Note that in the Hamiltonian cycle $y$, depending on whether $n$ is either even or odd, there are either two or $n$ different subsets of $\lfloor n / 2\rfloor$ disjoint edges in $y$. For each such maximum-size matching $\Gamma$ of $y$ and each $I \subset \Gamma$ of cardinality $k$, we define

$$
p_{I, \Gamma}=\prod_{\{i, j\} \in I} x_{i, j}
$$

In words, $p_{I, \Gamma}$ is the monomial corresponding to $k$ disjoint edges which are a subset of some maximum-size matching of $y$. Note that each $p_{I, \Gamma}$ takes on only values 0 or 1 . In order to use Lemma 1, we need to calculate

$$
\sum_{I, \Gamma} \sum_{x:\{i, j\} \in x} p_{I, \Gamma}(x)
$$

where in the first sum $\Gamma$ runs over all maximum-size matchings of $y$, and $I$ runs over all $k$-element subsets of $\Gamma$. We note that these polynomials were chosen with Lemma 1 in mind; namely so that for each edge $\{i, j\}$, (5) has only two different values: one value if $\{i, j\} \notin y$ and another value if $\{i, j\} \in y$.

Suppose that $n$ is even. Then $y$ has two maximum-size matchings, $\Gamma_{1}$ and $\Gamma_{2}$. Note that when we calculate (5), we are simply counting the number of Hamiltonian cycles containing both some $I \subset \Gamma_{\ell}$ of size $k$ and the edge $\{i, j\}$. Note that in each of $\Gamma_{1}$ and $\Gamma_{2}$, for each $i \in\{1,2, \ldots, n\}$, there is exactly one edge incident to vertex $i$. If $\{i, j\} \notin y$, the edge which is incident to $i$ and the edge which is incident to $j$ are distinct. If $\{i, j\} \in y$ then $\{i, j\}$ is in one of $\Gamma_{1}$ or $\Gamma_{2}$.

Let us pick some edge $\{i, j\} \notin y$. Then for each of the maximum-size matchings $\Gamma_{1}$ and $\Gamma_{2}$ there are $\left(\begin{array}{c}n / 2-2 \\ k-2\end{array}\right)$ subsets $I$ of size $k$ containing the edge incident to $i$ and the edge incident to $j$. For such subsets $I, I \cup\{i, j\}$ consists of $k-1$ distinct paths, $k-2$ of which are of length 1 , and one of which is of length 3 .

There are $2\left(\begin{array}{c}n / 2-2 \\ k-1\end{array}\right)$ subsets $I$ of size $k$ containing exactly one of the edges which are either incident to $i$ or to $j$. For such subsets $I, I \cup\{i, j\}$ consists of $k$ distinct paths, $k-1$ 
of which are of length 1 , one of which is of length 2 . Lastly, there are $\left(\begin{array}{c}n / 2-2 \\ k\end{array}\right)$ subsets $I$ of size $k$ containing neither the edge incident to $i$ nor the edge incident to $j$. For such subsets $I, I \cup\{i, j\}$ consists of $k+1$ distinct paths, each of length 1 . Thus, from Lemma 2 , we can see that if $\{i, j\} \notin y$ then we can calculate (5) (which we denote $f_{1}(n, k)$ ) to be

$$
\begin{aligned}
f_{1}(n, k)= & \sum_{I, \Gamma} \sum_{x:\{i, j\} \in x} p_{I, \Gamma}(x) \\
=2 & {\left[\left(\begin{array}{c}
n / 2-2 \\
k-2
\end{array}\right) 2^{k-2}(n-k-2) !+2\left(\begin{array}{c}
n / 2-2 \\
k-1
\end{array}\right) 2^{k-1}(n-k-2) !\right.} \\
& \left.\quad+\left(\begin{array}{c}
n / 2-2 \\
k
\end{array}\right) 2^{k}(n-k-2) !\right] .
\end{aligned}
$$

Recall that if $\{i, j\} \in y$, exactly one of $\Gamma_{1}$ or $\Gamma_{2}$ contains the edge $\{i, j\}$, say $\Gamma_{1}$ does. Then $\Gamma_{2}$ contains one edge incident to $i$, and a disjoint edge incident to $j$. By arguments similar to those above, and again using Lemma 2 , we can see that if $\{i, j\} \in y$ then we can calculate (5) (which we denote $f_{2}(n, k)$ ) to be

$$
\begin{aligned}
f_{2}(n, k)= & \sum_{I, \Gamma} \sum_{x:\{i, j\} \in x} p_{I, \Gamma}(x) \\
= & \left(\begin{array}{c}
n / 2-1 \\
k-1
\end{array}\right) 2^{k-1}(n-k-1) !+\left(\begin{array}{c}
n / 2-1 \\
k
\end{array}\right) 2^{k}(n-k-2) ! \\
& +\left(\begin{array}{c}
n / 2-2 \\
k-2
\end{array}\right) 2^{k-2}(n-k-2) !+2\left(\begin{array}{c}
n / 2-2 \\
k-1
\end{array}\right) 2^{k-1}(n-k-2) ! \\
& +\left(\begin{array}{c}
n / 2-2 \\
k
\end{array}\right) 2^{k}(n-k-2) !
\end{aligned}
$$

Thus, using these calculations and Lemma 1 , we see that if $n$ is even and $f \in P_{k}$ then

$$
-\frac{(n-1)}{2} \frac{f_{1}(n, k)}{f_{2}(n, k)-f_{1}(n, k)}=-\frac{n}{k}+1-\frac{n(k-1)}{k\left(n^{2}-k n-3 n+k+3\right)} \leq f(y) .
$$

Now suppose that $n$ is odd. Then $y$ has $n$ maximum-size matchings, $\Gamma_{1}, \ldots, \Gamma_{n}$, where $\Gamma_{i}$ does not have an edge incident to vertex $i$.

Note that for each $i \in\{1,2, \ldots, n\}$, and each $\Gamma_{\ell}, \ell \neq i$, there is exactly one edge incident to $i$. If $\{i, j\} \notin y$ and $i, j \neq \ell$, then in $\Gamma_{\ell}$ the edge incident to $i$ and the edge incident to $j$ are distinct.

If $\{i, j\} \in y$ then $\{i, j\}$ is in $(n-1) / 2$ of the $\Gamma_{j}$ 's. In $(n-1) / 2-1$ of the $\Gamma_{j}$ 's the edge incident to $i$ and the edge incident to $j$ are distinct. In $\Gamma_{j}$, there is only an edge incident to $i$, in $\Gamma_{i}$ there is only an edge incident to $j$.

Let us pick some edge $\{i, j\} \notin y$. Then for the maximum-size matchings $\Gamma_{\ell}, \ell \neq i, j$ there are $\left(\begin{array}{c}(n-1) / 2-2 \\ k-2\end{array}\right)$ subsets $I$ of size $k$ containing the edge incident to $i$ and the edge incident to $j$. For such subsets $I, I \cup\{i, j\}$ consists of $k-1$ disjoint paths, $k-2$ of which are of length 1 , one of which is of length 3 . There are $2\left(\begin{array}{c}(n-1) / 2-2 \\ k-1\end{array}\right)$ subsets $I$ of size $k$ containing exactly one of the edges which are either incident to $i$ or $j$. For such 
subsets $I, I \cup\{i, j\}$ consists of $k$ disjoint paths, $k-1$ of which are of length 1 , one of which is of length 2 . There are $\left(\begin{array}{c}(n-1) / 2-2 \\ k\end{array}\right)$ subsets $I$ of size $k$ containing neither the edge which incident to $i$ nor the edge incident to $j$. For such subsets $I, I \cup\{i, j\}$ consists of $k+1$ disjoint paths, each of length 1 .

In $\Gamma_{i}$ there are $\left(\begin{array}{c}(n-1) / 2-1 \\ k-1\end{array}\right)$ subsets $I$ of size $k$ containing the edge incident to $j(I \cup\{i, j\}$ consisting of $k-1$ paths of length 1 , one path of length 2$)$, and $\left(\begin{array}{c}(n-1) / 2-1 \\ k\end{array}\right)$ subsets $I$ of size $k$ not containing the edge incident to $j(I \cup\{i, j\}$ consisting of $k+1$ paths of length 1). Similarly, in $\Gamma_{j}$, there are $\left(\begin{array}{c}(n-1) / 2-1 \\ k-1\end{array}\right)$ subsets $I$ of size $k$ containing the edge incident to $i(I \cup\{i, j\}$ consisting of $k-1$ paths of length 1 , one path of length 2$)$, and $\left(\begin{array}{c}(n-1) / 2-1 \\ k\end{array}\right)$ subsets $I$ of size $k$ not containing the edge incident to $i(I \cup\{i, j\}$ consisting of $k+1$ paths of length 1 ). Recall that in calculating (5), we are simply counting the number of Hamiltonian cycles containing both some $I$ of size $k$ and the edge $\{i, j\}$. Thus, from Lemma 2, we can see that if $\{i, j\} \notin y$ then we can calculate (5) (which we denote $\left.g_{1}(n, k)\right)$ to be

$$
\begin{aligned}
& g_{1}(n, k)=\sum_{I, \Gamma} \sum_{x:\{i, j\} \in x} p_{I, \Gamma}(x) \\
& =(n-2)\left[\left(\begin{array}{c}
(n-1) / 2-2 \\
k-2
\end{array}\right) 2^{k-2}(n-k-2) !\right. \\
& +2\left(\begin{array}{c}
(n-1) / 2-2 \\
k-1
\end{array}\right) 2^{k-1}(n-k-2) ! \\
& \left.+\left(\begin{array}{c}
(n-1) / 2-2 \\
k
\end{array}\right) 2^{k}(n-k-2) !\right] \\
& +2\left[\left(\begin{array}{c}
(n-1) / 2-1 \\
k-1
\end{array}\right) 2^{k-1}(n-k-2)\right. \text { ! } \\
& \left.+\left(\begin{array}{c}
(n-1) / 2-1 \\
k
\end{array}\right) 2^{k}(n-k-2) !\right] .
\end{aligned}
$$

Recall that if $\{i, j\} \in y,(n-1) / 2$ of the $\Gamma_{\ell}$ 's contain the edge $\{i, j\},(n-1) / 2-1$ of the $\Gamma_{\ell}$ 's have the edge incident to $i$ and the edge incident to $j$ being distinct, $\Gamma_{j}$ does not have an edge incident to $j$ and $\Gamma_{i}$ does not have an edge incident to $i$. By arguments similar to those above, and again using Lemma 2, we find that for $\{i, j\} \in y$ we can calculate (5) (which we denote $g_{2}(n, k)$ ) to be

$$
\begin{aligned}
g_{2}(n, k)= & \sum_{I, \Gamma} \sum_{x:\{i, j\} \in x} p_{I, \Gamma}(x) \\
=(n-1) / 2 & {\left[\left(\begin{array}{c}
(n-1) / 2-1 \\
k-1
\end{array}\right) 2^{k-1}(n-k-1) !\right.} \\
& \left.+\left(\begin{array}{c}
(n-1) / 2-1 \\
k
\end{array}\right) 2^{k}(n-k-2) !\right]
\end{aligned}
$$




$$
\begin{aligned}
& +((n-1) / 2-1)\left[\left(\begin{array}{c}
(n-1) / 2-2 \\
k-2
\end{array}\right) 2^{k-2}(n-k-2) !\right. \\
& +2\left(\begin{array}{c}
(n-1) / 2-2 \\
k-1
\end{array}\right) 2^{k-1}(n-k-2) ! \\
& \left.+\left(\begin{array}{c}
(n-1) / 2-2 \\
k
\end{array}\right) 2^{k}(n-k-2) !\right] \\
& +2\left[\left(\begin{array}{c}
(n-1) / 2-1 \\
k-1
\end{array}\right) 2^{k-1}(n-k-2) !\right. \\
& +\left(\begin{array}{c}
(n-1) / 2-1 \\
k
\end{array}\right) 2^{k}(n-k-2) !
\end{aligned}
$$

Thus, using these calculations and Lemma 1, we see that if $n$ is odd and $f \in P_{k}$ then

$$
-\frac{(n-1)}{2} \frac{g_{1}(n, k)}{g_{2}(n, k)-g_{1}(n, k)}=-\frac{n}{k}+1-\frac{n(k-1)}{k\left(n^{2}-n k-4 n+4+2 k\right)} \leq f(y) .
$$

Now we can prove Theorem 1:

Proof of Theorem 1. Recall that we assume $n \geq 9$ and $\lfloor n / 2\rfloor \geq k$. Note that both

$$
\frac{n(k-1)}{k\left(n^{2}-k n-3 n+k+3\right)} \quad \text { and } \quad \frac{n(k-1)}{k\left(n^{2}-n k-4 n+4+2 k\right)}
$$

are bounded above in absolute value by $10 / n$. Thus, from Proposition 1 we know that for $a_{k}=n / k+10 / n$, if $f \in P_{k}$, then for each $y \in X$, we have $-a_{k}+1 \leq f(y)$. This implies that $\left(f+\left(a_{k}-1\right) 1\right)(y) \geq 0$ for all $y \in X$. It is clear that $f+\left(a_{k}-1\right) 1$ has average value

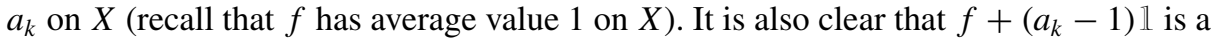
linear function on $X$ (recall that $f$ is linear; the function 1 corresponds to inner product with the vector $(2 / n, 2 / n, \ldots, 2 / n))$. Thus, we have $f+\left(a_{k}-1\right) 1 \in a_{k} Q$. Thus, we have

$$
Q-1 \subset P_{k}-1 \subset a_{k}\left(Q-q_{1}\right)
$$

\section{Acknowledgements}

The author thanks Alexander Barvinok for his helpful ideas and encouragement, see Section 1.2.

\section{References}

1. Barvinok, A.: Personal communication.

2. Goemans, M.X.: Worst-case comparison of valid inequalities for the TSP. Math. Programming 69(2, Ser. A), 335-349 (1995). 
3. Grötschel, M., Padberg, M.W.: On the symmetric travelling salesman problem. I. Inequalities. Math. Programming 16(3), 265-280 (1979).

4. M. Grötschel, L.L., Schrijver, A.: Geometric Algorithms and Combinatorial Optimization. Algorithms and Combinatorics, vol. 2. Springer, Berlin (1988).

5. Karp, R., Papadimitriou, C.: On linear characterizations of combinatorial optimization problems. In: Proceedings of the 21st Annual Symposium on Foundations of Computer Science, pp. 1-9 (1980).

6. Kojima, M., Tunçel, L.: Cones of matrices and successive convex relaxations of nonconvex sets. SIAM J. Optim. 10(3), 750-778 (electronic) (2000).

7. Lasserre, J.: An explicit exact SDP relaxation for nonlinear 0-1 programs. In: K. Aardal, A. Gerards (eds.) Integer Programming and Combinatorial Optimization 2001, pp. 293-303. No. 2081 in Lecture Notes in Computer Science. Springer, Berlin (2001).

8. Laurent, M.: A comparison of the Sherali-Adams, Lovász-Schrijver, and Lasserre relaxations for 0-1 programming. Math. Oper. Res. 28(3), 470-496 (2003).

9. Lovász, L., Schrijver, A.: Cones of matrices and set-functions and 0-1 optimization. SIAM J. Optim. 12, 345-375 (1991).

10. Schrijver, A.: Combinatorial Optimization: Polyhedra and Efficiency. Algorithms and Combinatorics, 24, vol. B. Springer, Berlin (2003).

11. Sherali, H., Adams, W.: A hierarchy of relaxations between the continuous and convex hull representations for zero-one programming problems. SIAM J. Discrete Math. 3, 411-430 (1990).

12. Veomett, E.: A positive semidefinite approximation of the symmetric traveling salesman polytope. URL http://arxiv.org/pdf/math.CO/0610193.

Received March 22, 2006, and in revised form January 24, 2007. Online publication May 18, 2007. 